\title{
Increase in extrapulmonary tuberculosis in England and Wales 1999-2006
}

\author{
M E Kruijshaar, ${ }^{1}$ | Abubakar ${ }^{1,2}$
}

${ }^{1}$ Tuberculosis Section, Respiratory and Systemic Infections Department, Centre for Infections, Health Protection Agency, London, UK; ${ }^{2}$ School of Medicine, Health Policy and Practice, University of East Anglia, Norwich, UK

Correspondence to:

Dr M E Kruiishaar, Tuberculosis

Section, Respiratory and Systemic Infections Department, Centre for Infections, Health Protection Agency, 61 Colindale Avenue, London NW9 5E0, UK: michelle.kruijshaar@hpa.org.uk

Received 17 April 2009 Accepted 22 September 2009 Published Online First 22 October 2009

\begin{abstract}
Background: Extrapulmonary tuberculosis appears to be increasing in England and Wales. The trends in extrapulmonary tuberculosis and factors associated with these trends were examined.

Methods: National tuberculosis surveillance data from 1999-2006 for England and Wales were used, including demographic, clinical and laboratory information. Trends in the proportion of tuberculosis cases with extrapulmonary disease were investigated using the $\chi^{2}$ trend test and associated factors using logistic regression.

Results: Among all the cases of tuberculosis, the proportion with extrapulmonary disease increased from $48 \%$ in 1999 (2717 cases) to 53\% in 2006 (4205 cases, $p<0.001)$. Regression analysis showed that the rise in extrapulmonary disease was associated with an increase in the proportion of non-UK born cases (odds ratio 2.7, $95 \% \mathrm{Cl} 2.6$ to 2.8). A more than threefold increase was observed in the proportion of all tuberculosis cases with miliary tuberculosis from $0.7 \%$ of all cases (38 cases) to $2.2 \%$ (180 cases, $p<0.001$ ). This rise was not associated with changes in place of birth or in any of the other risk factors identified.

Conclusions: The proportion of cases with extrapulmonary disease has increased over the study period. To a large extent this is due to an increasing proportion of nonUK born cases. Reasons for the rise in miliary tuberculosis require further investigation. Clinicians should have a higher index of clinical suspicion of extrapulmonary tuberculosis in non-UK born cases.
\end{abstract}

Tuberculosis remains a major global public health issue. ${ }^{1}$ The most common form of the disease is tuberculosis of the lungs, but it can affect almost any part of the body including lymph nodes, gastrointestinal tract, bones and joints, genitourinary tract and central nervous system, and it may affect multiple organs. ${ }^{2}$ Miliary disease and meningitis are associated with particularly poor outcomes. $^{3}$

The diagnosis and management of extrapulmonary tuberculosis poses particular challenges. The diagnosis is often difficult owing to the wide spectrum of clinical presentations, the limited specificity of the manifestations and difficulties in obtaining specimens for culture. ${ }^{2}$ It is frequently made using histological or radiological evidence in combination with signs or symptoms, but can only be confirmed by microbiological culture. ${ }^{4}$ Managing cases with extrapulmonary tuberculosis is complicated owing to the sometimes longer course of treatment $t^{4}$ and difficulties in monitoring progress in the absence of follow-up samples.

Many industrialised nations have noted an increase in the proportion of cases presenting with extrapulmonary tuberculosis. ${ }^{5-7}$ In the USA the proportion increased from $16 \%$ in 1993 to $21 \%$ in $2006 .{ }^{8}$ Recent reports from the Netherlands and Canada suggest that it now affects nearly $40 \%$ of tuberculosis cases. ${ }^{9}{ }^{10}$ Changes in the demographic characteristics of tuberculosis cases and the HIV epidemic are thought to be responsible for this increase. ${ }^{67}$ Factors associated with extrapulmonary tuberculosis include female gender, ${ }^{11-14}$ young age, ${ }^{11-13}{ }^{15}$ Asian and African origin ${ }^{2114-16}$ and HIV infection. ${ }^{2} 12141517$

In the UK, more than $40 \%$ of cases reported currently have extrapulmonary tuberculosis (without pulmonary involvement). ${ }^{18}$ Nevertheless, the trends and the factors associated with any changes have not been investigated. We therefore analysed national surveillance data to examine recent trends in extrapulmonary tuberculosis in England and Wales between 1999 and 2006 and explored which factors could be responsible for this increase.

\section{METHODS \\ Data sources and record linkage}

In England and Wales, clinical and demographic information on tuberculosis cases are reported voluntarily to the Enhanced Tuberculosis Surveillance (ETS) system. All cases reported between 1 January 1999 and 31 December 2006 were included in the analysis. Treatment outcome is collected for cases reported to the ETS 1 year after the start of treatment and is available for cases reported between 2000 and 2005. Drug susceptibility testing results for culture-confirmed cases are reported through the UK Mycobacterial Surveillance Network (MycobNet) and matched to case reports annually. Information on HIV status was obtained by matching to the national HIV/ AIDS reports database, as described previously. ${ }^{19} 20$

\section{Definitions}

The case definition for national surveillance includes culture-confirmed cases with a positive culture of Mycobacterium tuberculosis complex (including $M$ tuberculosis, $M$ bovis and $M$ africanum), and other than culture-confirmed cases who, in the absence of culture confirmation, meet the following criteria: (1) clinician's judgement that the patient's clinical and/or radiological signs and/or symptoms are compatible with tuberculosis, and (2) decision to treat the patient with a full course of antituberculosis treatment.

The following sites of disease are distinguished in the ETS: pulmonary (tuberculosis affecting the lung parenchyma), bone/joint, cryptic disseminated, extrathoracic lymph nodes, gastrointestinal, genitourinary, intrathoracic lymph nodes, menin- 
gitis, miliary, pleural, spinal tuberculosis, other extrapulmonary and unknown. If any site other than pulmonary or unknown was involved, cases were considered to have "extrapulmonary tuberculosis".

To investigate the effect of migration and time since migration, a composite variable "place of birth/time since entry" was created differentiating between UK born and nonUK born, and subdividing non-UK born by time since entry. Ethnic groups were based on the Office of National Statistics classifications. $^{21}$

\section{Analysis of data}

For each site of disease, the number and proportion of new tuberculosis cases were tabulated by year of reporting. Trends in the proportion of cases by site of disease were assessed using the $\chi^{2}$ trend test. Numbers and proportions were also tabulated by potential explanatory variables which were selected based on previous literature (age group, gender, place of birth/time since entry, ethnic group, region of reporting, previous diagnosis and HIV status). Proportions may not always add up to $100 \%$ owing to missing information.

Factors associated with extrapulmonary tuberculosis were investigated using univariable and multivariable logistic regression models comparing all cases with extrapulmonary involvement to exclusively pulmonary cases using Stata 10. Factors explored were the potential explanatory variables and year of reporting (to assess the trend). Multivariable models were built using a forward-fitting approach (inclusion for $\mathrm{p}<0.2$ ). Interactions with place of birth/time since entry were assessed (for $p<0.01$ ). Analyses were repeated for extrapulmonary sites showing substantial increases comparing all cases with the respective site of disease to those without.

\section{RESULTS}

\section{Study population}

A total of 55607 cases of tuberculosis were reported in England and Wales between 1999 and 2006. Of these, 50\% $(n=27762)$ had exclusive pulmonary tuberculosis and $41 \%(n=22$ 933) had exclusive extrapulmonary disease. In $8 \%$ of cases $(n=4341)$ both sites were involved and in $1 \%(n=571)$ the site was unknown. Common extrapulmonary sites included extrathoracic lymph nodes, the pleura and intrathoracic lymph nodes, which were affected in 18\%, $8 \%$ and $7 \%$ of all tuberculosis cases reported, respectively.

The overall median age of all tuberculosis cases was 36 years, $55 \%$ were male, $60 \%$ non-UK born, $25 \%$ of white ethnic group, $7 \%$ had a previous diagnosis, $5 \%$ were known to be HIV coinfected and 59\% were culture-confirmed (table 1). The median age of cases with extrapulmonary disease was 35 years, $51 \%$ were male, $19 \%$ were UK born, $13 \%$ of white ethnic group, $6 \%$ had a previous diagnosis, $5 \%$ were known to be HIV co-infected and $53 \%$ of cases were culture-confirmed. Cases with only pulmonary disease were more often male $(58 \%)$, born in the UK (37\%), of white ethnic group (36\%), culture-confirmed $(66 \%)$ and more often had a previous diagnosis (9\%).

\section{Trends by site of disease}

The total number of cases with extrapulmonary and pulmonary tuberculosis increased between 1999 and 2006 (table 2). The proportion of all cases that had extrapulmonary disease (with and without pulmonary involvement) rose from 48\% in 1999 to $53 \%$ in $2006(\mathrm{p}<0.001)$
The largest increase was seen in miliary tuberculosis where the proportion rose threefold from $0.7 \%$ to $2.2 \%(p<0.001)$. Tuberculosis meningitis, gastrointestinal and spinal tuberculosis also rose considerably compared with all tuberculosis cases $(p<0.001, p=0.002$ and $p=0.007$, respectively). A small decrease in laryngeal tuberculosis was observed $(p=0.005)$.

\section{Factors associated with the increase in extrapulmonary disease} We explored the trend in extrapulmonary disease further using logistic regression to investigate possible explanatory factors. Univariable analysis showed a statistically significant increased risk of tuberculosis cases presenting with extrapulmonary disease in 2004, 2005 and 2006 compared with 1999 (table 3). The odds ratios increased for each consecutive year since 2001.

On multivariable analysis, extrapulmonary cases were more likely to be female (odds ratio (OR) 1.27, 95\% CI 1.21 to 1.32), born abroad with entry into the UK more than a year before diagnosis $(p<0.001)$ and of non-white ethnic group $(p<0.001)$ compared with pulmonary cases (table 3 ). They were also less likely to have had a previous diagnosis of tuberculosis (OR 0.72 (95\% CI 0.67 to 0.78$)$, while HIV co-infection was not associated with extrapulmonary site of disease $(p=0.687)$.

After correcting for gender, age, place of birth/time since entry, ethnic group, region of reporting and previous diagnosis, there was no longer an increased risk of extrapulmonary disease in any year compared with 1999 (see multivariable model in table 3). Further exploration revealed that correcting for being non-UK born without adjusting for any of the other variables made the increase in extrapulmonary disease statistically nonsignificant.

Statistically significant interactions were found between place of birth/time since entry and ethnic group, previous diagnosis, age and sex. Inclusion of these interactions in the model altered the effect of year of reporting by less than $0.5 \%$ and was therefore not presented here.

Subgroup analyses showed that the proportion of extrapulmonary cases did not increase in most subgroups such as the UK born, non-UK born, recent entrants and the black African ethnic group. There was, however, a significant increase in the Indian, Pakistani, Bangladeshi ethnic group and in cases who entered the UK 5-10 years before being diagnosed with tuberculosis.

Culture confirmation of extrapulmonary cases increased from $51 \%$ in 1999 to $56 \%$ in 2006 . When analyses were repeated among culture-confirmed cases only, the increase was smaller but still statistically significant for 2005 and 2006 (OR 1.11, 95\% CI 1.01 to 1.21 and $1.17,95 \%$ CI 1.07 to 1.28 , respectively).

\section{Miliary tuberculosis and tuberculosis meningitis}

Owing to the considerable increase in miliary tuberculosis, factors associated with this site of disease were explored further (table 4). Cases with miliary tuberculosis were more likely to be over 60 years of age (OR 1.71, 95\% CI 1.30 to 2.25), born abroad with entry into the UK more than a year before diagnosis $(p=0.008-0.050)$, of Indian, Pakistani, Bangladeshi ethnic group (OR 1.92, 95\% CI 1.36 to 2.71 ) and co-infected with HIV (OR 4.48, 95\% CI 3.43 to 5.84); and less likely to have had a previous diagnosis of tuberculosis (OR $0.60,95 \%$ CI 0.42 to 0.86) compared with all other cases of tuberculosis. The increasing trend remained statistically significant for all years after correcting for sex, age, place of birth/time since entry, ethnic group, region of reporting, previous diagnosis and HIV co-infection. A similar increase was noted when the analysis was restricted to culture-confirmed cases. 
Table 1 Demographic and clinical characteristics of tuberculosis cases reported in England and Wales, 1999-2006

\begin{tabular}{|c|c|c|c|c|c|c|}
\hline & \multicolumn{2}{|c|}{ All cases $(N=55607)$} & \multicolumn{2}{|c|}{$\begin{array}{l}\text { Extrapulmonary site } \\
(\mathrm{N}=27274)\end{array}$} & \multicolumn{2}{|c|}{$\begin{array}{l}\text { Only pulmonary site } \\
\text { ( } N=27762 \text { ) }\end{array}$} \\
\hline & $\mathbf{N}$ & $\%$ & $\mathbf{N}$ & $\%$ & $\mathbf{N}$ & $\%$ \\
\hline Gender (male) & 30380 & 55 & 13877 & 51 & 16220 & 58 \\
\hline Median age (IOR) & \multicolumn{2}{|c|}{$36(26-55)$} & \multicolumn{2}{|c|}{$35(26-50)$} & \multicolumn{2}{|c|}{$37(26-60)$} \\
\hline UK born ( $11 \%$ unknown) & 15658 & 28 & 5176 & 19 & 10358 & 37 \\
\hline \multicolumn{7}{|l|}{ Ethnic group } \\
\hline White & 14042 & 25 & 3871 & 14 & 10091 & 36 \\
\hline Black African & 11412 & 21 & 6095 & 22 & 5225 & 19 \\
\hline $\begin{array}{l}\text { Indian, Pakistani, } \\
\text { Bangladeshi }\end{array}$ & 20212 & 36 & 12395 & 45 & 7613 & 27 \\
\hline Other & 7269 & 13 & 3646 & 13 & 3560 & 13 \\
\hline Reported in London & 23671 & 43 & 12291 & 45 & 11156 & 40 \\
\hline Previous TB diagnosis* & 3985 & 7 & 1568 & 6 & 2384 & 9 \\
\hline HIV co-infection $\dagger$ & 2602 & 5 & 1259 & 5 & 1314 & 5 \\
\hline Culture confirmation & 32804 & 59 & 14351 & 53 & 18453 & 66 \\
\hline
\end{tabular}

*20\% unknown.

†For cases reported between 1999 and 2005.

$\mathrm{IQR}$, interquartile range; TB, tuberculosis.

\section{DISCUSSION}

The rise in extrapulmonary tuberculosis cases between 1999 and 2006 exceeded the increase in the number of cases with pulmonary disease. With $43 \%$ of cases presenting with exclusive extrapulmonary disease in 2006, England now has one of the highest proportions of extrapulmonary disease among western countries. ${ }^{5-10}$ The proportion of cases with miliary tuberculosis increased more than threefold.

\section{Extrapulmonary disease}

Multivariable analysis showed that the increasing proportion of extrapulmonary cases could be explained by the growing proportion of non-UK born cases, a finding that is consistent with previous studies. ${ }^{7116}$ One possible explanation for the increasing risk of extrapulmonary tuberculosis as a result of a rise in non-UK born cases could be an effect of "time since infection". In the presence of widespread active transmission in tuberculosis endemic settings, the majority of new cases present with pulmonary disease. In contrast, many migrants acquired their infection prior to arrival in the UK and subsequently present with reactivation disease. This is consistent with the observation by Musellim et al that extrapulmonary disease was more likely to develop 5 or more years after contact, while pulmonary disease was more likely to develop early. ${ }^{22}$ In our study the odds ratios for extrapulmonary disease among the non-UK born increased with time since arrival, indicating that recent entrants, who are likely to have been infected more recently than those who arrived in the country a longer time ago, presented less often with extrapulmonary disease. This supports the notion that reactivation disease is more likely to

Table 2 Number and proportion of tuberculosis cases reported with pulmonary and extrapulmonary tuberculosis, England and Wales 1999 and 2006

\begin{tabular}{|c|c|c|c|c|c|c|}
\hline & \multicolumn{2}{|l|}{1999} & \multicolumn{2}{|l|}{2006} & \multirow{2}{*}{$\begin{array}{l}\% \text { Increase } \\
\text { in } \mathrm{N}\end{array}$} & \multirow{2}{*}{$\begin{array}{l}\text { p Value } \\
\left(\chi^{2} \text { trend }\right)^{*}\end{array}$} \\
\hline & $\mathbf{N}$ & $\%(95 \% \mathrm{CI})$ & $\mathbf{N}$ & $\%(95 \% \mathrm{Cl})$ & & \\
\hline ET lymph nodes & 1066 & 19 (18 to 20$)$ & 1575 & $20(19$ to 21$)$ & $47.7 \%$ & $<0.001$ \\
\hline Pleural & 436 & $7.6(7.0$ to 8.4$)$ & 641 & $8.0(7.4$ to 8.6$)$ & $47.0 \%$ & 0.041 \\
\hline IT lymph nodes & 371 & 6.5 (5.9 to 7.2$)$ & 591 & $7.3(6.8$ to 7.9$)$ & $59.3 \%$ & 0.001 \\
\hline Gastrointestinal & 175 & 3.1 (2.7 to 3.6$)$ & 315 & $3.9(3.5$ to 4.4$)$ & $80.0 \%$ & 0.002 \\
\hline Spine & 145 & $2.5(2.2$ to 3.0$)$ & 268 & $3.3(3.0$ to 3.7$)$ & $84.8 \%$ & 0.007 \\
\hline Bone (not spine) & 140 & $2.5(2.1$ to 2.9$)$ & 161 & $2.0(1.7$ to 2.3$)$ & $15.0 \%$ & $<0.001$ \\
\hline Genitourinary & 111 & $1.9(1.6$ to 2.3$)$ & 135 & 1.7 (1.4 to 2.0$)$ & $21.6 \%$ & 0.008 \\
\hline Miliary TB & 38 & $0.7(0.5$ to 0.9$)$ & 180 & $2.2(1.9$ to 2.6$)$ & $373.7 \%$ & $<0.001$ \\
\hline TB meningitis & 86 & $1.5(1.2$ to 1.9$)$ & 165 & $2.0(1.8$ to 2.4$)$ & $91.9 \%$ & $<0.001$ \\
\hline CNS other & 41 & $0.7(0.5$ to 1.0$)$ & 70 & $0.9(0.7$ to 1.1$)$ & $70.7 \%$ & 0.240 \\
\hline Cryptic disseminated & 31 & $0.5(0.4$ to 0.8$)$ & 38 & $0.5(0.3$ to 0.6$)$ & $22.6 \%$ & 0.075 \\
\hline Laryngeal & 15 & $0.3(0.2$ to 0.4$)$ & 13 & $0.2(0.1$ to 0.3$)$ & $-13.3 \%$ & 0.005 \\
\hline Extrapulmonary only & 2310 & 41 (39 to 42 ) & 3480 & 43 (42 to 44$)$ & $50.6 \%$ & $<0.001$ \\
\hline $\begin{array}{l}\text { Extrapulmonary with } \\
\text { pulmonary involvement }\end{array}$ & 407 & $7.1(6.5$ to 7.8$)$ & 725 & $9.0(8.4$ to 9.7$)$ & $78.1 \%$ & $<0.001$ \\
\hline Pulmonary only & 2923 & 51 (50 to 53 ) & 3742 & 47 (45 to 48 ) & $28.0 \%$ & $<0.001$ \\
\hline All TB & 5704 & 100 & 8051 & 100 & $41.1 \%$ & Ref \\
\hline
\end{tabular}

${ }^{*} \chi^{2}$ trend test with one degree of freedom for changes across each year.

$95 \% \mathrm{Cl}, 95 \%$ confidence interval for proportions using Wilson procedure; ET, extrathoracic; IT, intrathoracic; TB, tuberculosis; extrapulmonary only, all extrapulmonary tuberculosis sites without pulmonary involvement; pulmonary only, pulmonary tuberculosis without extrapulmonary involvement; all TB, all tuberculosis cases including those with unknown or other extrapulmonary sites of disease; Ref, reference group. 
Table 3 Univariable and multivariable analysis for the association between characteristics of tuberculosis cases and extrapulmonary site of disease

\begin{tabular}{|c|c|c|c|c|c|}
\hline & \multicolumn{2}{|c|}{ Number } & \multirow{2}{*}{$\begin{array}{l}\text { Univariable } \\
\text { OR (95\% CI) }\end{array}$} & \multicolumn{2}{|l|}{ Multivariable* } \\
\hline & EP & All & & OR (95\% CI) & p Value \\
\hline \multicolumn{6}{|l|}{ Year } \\
\hline 1999 & 2717 & 5704 & Reference & Reference & 0.001 \\
\hline 2000 & 2966 & 6271 & $0.98(0.91$ to 1.05$)$ & $1.00(0.92$ to 1.09$)$ & \\
\hline 2001 & 3058 & 6597 & 0.95 (0.88 to 1.02$)$ & $0.95(0.87$ to 1.03$)$ & \\
\hline 2002 & 3181 & 6794 & $0.98(0.91$ to 1.05$)$ & $0.89(0.81$ to 0.97$)$ & \\
\hline 2003 & 3359 & 6913 & $1.03(0.96$ to 1.11$)$ & $0.95(0.87$ to 1.03$)$ & \\
\hline 2004 & 3600 & 7240 & 1.09 (1.01 to 1.16$)$ & $1.01(0.92$ to 1.10$)$ & \\
\hline 2005 & 4188 & 8037 & 1.19 (1.11 to 1.27$)$ & $1.03(0.95$ to 1.12$)$ & \\
\hline 2006 & 4205 & 8051 & 1.21 (1.13 to 1.29$)$ & $1.06(0.97$ to 1.15$)$ & \\
\hline \multicolumn{6}{|l|}{ Gender } \\
\hline Male & 13877 & 30380 & Reference & Reference & $<0.001$ \\
\hline Female & 13343 & 25121 & $1.36(1.31$ to 1.40$)$ & $1.27(1.21$ to 1.32$)$ & \\
\hline \multicolumn{6}{|l|}{ Age group } \\
\hline $0-14$ & 1502 & 3209 & $0.90(0.84$ to 0.97$)$ & $1.18(1.08$ to 1.30$)$ & \\
\hline $15-29$ & 8219 & 16391 & Reference & Reference & $<0.001$ \\
\hline $30-44$ & 8730 & 15732 & 1.25 (1.19 to 1.30$)$ & $1.39(1.32$ to 1.47$)$ & \\
\hline $45-59$ & 4212 & 8570 & $0.97(0.92$ to 1.02$)$ & $1.32(1.23$ to 1.42$)$ & \\
\hline $60+$ & 4602 & 11690 & 0.64 (0.61 to 0.68$)$ & $1.06(0.99$ to 1.14$)$ & \\
\hline \multicolumn{6}{|l|}{ Place of birth } \\
\hline Born in UK & 5176 & 15658 & Reference & Reference & $<0.001$ \\
\hline Born abroad, entry $<1$ year ago & 1006 & 2757 & 1.17 (1.07 to 1.27$)$ & $0.74(0.67$ to 0.83$)$ & \\
\hline Born abroad, entry $1-2$ years ago & 2191 & 3818 & 2.72 (2.53 to 2.92$)$ & $1.67(1.52$ to 1.83$)$ & \\
\hline Born abroad, entry $2-5$ years ago & 4644 & 7583 & 3.21 (3.03 to 3.40$)$ & $1.90(1.75$ to 2.05$)$ & \\
\hline Born abroad, entry $5-10$ years ago & 2719 & 4497 & $3.11(2.91$ to 3.34$)$ & $1.75(1.60$ to 1.92$)$ & \\
\hline Born abroad, entry $10+$ years ago & 5350 & 9134 & $2.90(2.75$ to 3.06$)$ & $1.48(1.37$ to 1.60$)$ & \\
\hline Born abroad, year entry missing & 3185 & 5773 & $2.54(2.38$ to 2.70$)$ & $1.43(1.32$ to 1.56$)$ & \\
\hline \multicolumn{6}{|l|}{ Ethnic group } \\
\hline White & 3871 & 14042 & Reference & Reference & $<0.001$ \\
\hline Black African & 6095 & 11412 & $3.04(2.89$ to 3.20$)$ & 1.99 (1.83 to 2.17$)$ & \\
\hline Indian, Pakistani, Bangladeshi & 12395 & 20212 & 4.24 (4.05 to 4.45$)$ & $3.09(2.87$ to 3.32$)$ & \\
\hline Other & 3646 & 7269 & 2.67 (2.52 to 2.83 ) & $1.88(1.73$ to 2.05$)$ & \\
\hline \multicolumn{6}{|l|}{ Region of reporting } \\
\hline Other & 14983 & 31936 & Reference & Reference & 0.092 \\
\hline London & 12291 & 23671 & $1.22(1.18$ to 1.26$)$ & 1.04 (0.99 to 1.09$)$ & \\
\hline \multicolumn{6}{|l|}{ Previous TB diagnosis } \\
\hline No & 20163 & 40287 & Reference & Reference & $<0.001$ \\
\hline Yes & 1568 & 3985 & 0.65 (0.60 to 0.69$)$ & 0.72 (0.67 to 0.78$)$ & \\
\hline \multicolumn{6}{|l|}{ HIV co-infection† } \\
\hline No/unknown & 26015 & 53005 & Reference & & \\
\hline Yes & 1259 & 2602 & $1.00(0.92$ to 1.08$)$ & & \\
\hline
\end{tabular}

* Overall $\mathrm{p}$ value for categorical variables.

$\dagger$ For cases reported between 1999 and 2005.

$\mathrm{EP}$, extrapulmonary, $\mathrm{OR}$, odds ratio; TB, tuberculosis.

present at an extrapulmonary site. The subgroup analysis showing a rise in extrapulmonary disease in the Indian, Pakistani, Bangladeshi ethnic group also supports this. This group is known to include a large proportion of migrants who arrived in the country many years ago. The observed trend may thus be driven by an increase among longer-term migrants with reactivation disease.

Alternative explanations have been proposed for the increasing trend of extrapulmonary tuberculosis among immigrants to the UK including differences in the genetic make-up of the host's immune system, as well as the strain of organism prevalent in certain parts of the world. Further research is needed to investigate the role of these factors.

In contrast to other studies, we did not find an association between the rise in extrapulmonary tuberculosis and HIV coinfection. ${ }^{12} 141523$ It is unlikely that an increase in HIV coinfection would have contributed significantly to the trend since these patients constituted a relatively small proportion of all cases. An alternative explanation for the lack of association may be due to the inclusion of a substantial number of cases with pleural tuberculosis as an extrapulmonary site in this study. As an inverse association has been reported between HIV co-infection and pleural tuberculosis, ${ }^{12}$ this may obscure an association.

Increased awareness of tuberculosis may also lead to an increase in extrapulmonary disease, as it could result in more clinical diagnoses being made in the absence of culture confirmation. This is, however, unlikely as an increasing trend was observed among culture-confirmed cases.

\section{Miliary tuberculosis}

Although most of the risk factors for extrapulmonary tuberculosis were also found to be associated with miliary tuberculosis, they could not explain the observed increase. In line with 
Table 4 Univariable and multivariable analysis for miliary tuberculosis among tuberculosis cases

\begin{tabular}{|c|c|c|c|c|c|}
\hline & \multicolumn{2}{|l|}{ Number } & \multirow{2}{*}{$\begin{array}{l}\text { Univariable } \\
\text { OR (95\% Cl) }\end{array}$} & \multicolumn{2}{|l|}{ Multivariable* } \\
\hline & Miliary & All & & OR $(95 \% \mathrm{CI})$ & p Value \\
\hline \multicolumn{6}{|l|}{ Year } \\
\hline 1999 & 38 & 5704 & Reference & Reference & $<0.001$ \\
\hline 2000 & 100 & 6271 & 2.40 (1.65 to 3.50$)$ & 2.75 (1.69 to 4.47$)$ & \\
\hline 2001 & 106 & 6597 & 2.43 (1.68 to 3.53 ) & $3.11(1.93$ to 5.01$)$ & \\
\hline 2002 & 128 & 6794 & $2.88(2.00$ to 4.14$)$ & $3.33(2.08$ to 5.33$)$ & \\
\hline 2003 & 127 & 6913 & 2.78 (1.93 to 4.00$)$ & $3.54(2.21$ to 5.65$)$ & \\
\hline 2004 & 154 & 7240 & 3.24 (2.27 to 4.62$)$ & $4.19(2.65$ to 6.65$)$ & \\
\hline 2005 & 163 & 8037 & 3.08 (2.16 to 4.39$)$ & 3.47 (2.19 to 5.51$)$ & \\
\hline 2006 & 180 & 8051 & $3.42(2.40$ to 4.86$)$ & & \\
\hline \multicolumn{6}{|l|}{ Gender } \\
\hline Male & 506 & 30380 & Reference & Reference & 0.162 \\
\hline Female & 490 & 25121 & 1.18 (1.04 to 1.33$)$ & $1.13(0.95$ to 1.33$)$ & \\
\hline \multicolumn{6}{|l|}{ Age group } \\
\hline $0-14$ & 40 & 3209 & 0.85 (0.61 to 1.19 ) & $1.04(0.66$ to 1.65$)$ & \\
\hline $15-29$ & 244 & 16391 & Reference & Reference & 0.003 \\
\hline $30-44$ & 309 & 15732 & $1.33(1.12$ to 1.57$)$ & $1.12(0.90$ to 1.41$)$ & \\
\hline $45-59$ & 144 & 8570 & $1.13(0.92$ to 1.40$)$ & $1.14(0.85$ to 1.53$)$ & \\
\hline $60+$ & 259 & 11690 & $1.50(1.26$ to 1.79$)$ & $1.71(1.30$ to 2.25$)$ & \\
\hline \multicolumn{6}{|l|}{ Place of birth } \\
\hline Born in UK & 161 & 15658 & Reference & Reference & 0.091 \\
\hline Born abroad, entry < 1 year ago & 57 & 2757 & 2.03 (1.50 to 2.76$)$ & $1.22(0.78$ to 1.92$)$ & \\
\hline Born abroad, entry $1-2$ years ago & 82 & 3818 & 2.10 (1.61 to 2.75$)$ & $1.49(1.00$ to 2.23$)$ & \\
\hline Born abroad, entry $2-5$ years ago & 159 & 7583 & 2.06 (1.65 to 2.56$)$ & $1.43(1.00$ to 2.05$)$ & \\
\hline Born abroad, entry $5-10$ years ago & 76 & 4497 & 1.65 (1.26 to 2.18$)$ & $1.19(0.79$ to 1.80$)$ & \\
\hline Born abroad, entry $10+$ years ago & 205 & 9134 & 2.22 (1.80 to 2.73$)$ & 1.59 (1.13 to 2.24$)$ & \\
\hline Born abroad, year entry missing & 142 & 5773 & 2.44 (1.94 to 3.07$)$ & $1.63(1.13$ to 2.35$)$ & \\
\hline \multicolumn{6}{|l|}{ Ethnic group } \\
\hline White & 143 & 14042 & Reference & Reference & $<0.001$ \\
\hline Black African & 263 & 11412 & 2.30 (1.87 to 2.82 ) & 1.34 (0.90 to 1.99$)$ & \\
\hline Indian, Pakistani, Bangladeshi & 415 & 20212 & 2.05 (1.69 to 2.48 ) & $1.92(1.36$ to 2.71$)$ & \\
\hline Other & 125 & 7269 & 1.71 (1.34 to 2.17 ) & $1.34(0.90$ to 2.00$)$ & \\
\hline \multicolumn{6}{|l|}{ Region of reporting } \\
\hline Other & 601 & 31936 & Reference & & \\
\hline London & 395 & 23671 & 0.88 (0.78 to 1.00$)$ & & \\
\hline \multicolumn{6}{|l|}{ Previous TB diagnosis } \\
\hline No & 712 & 40287 & Reference & Reference & 0.003 \\
\hline Yes & 41 & 3985 & 0.58 (0.42 to 0.79 ) & $0.60(0.42$ to 0.86$)$ & \\
\hline \multicolumn{6}{|l|}{ HIV co-infection† $\dagger$} \\
\hline Nounknown & 670 & 53005 & Reference & Reference & $<0.001$ \\
\hline Yes & 146 & 2602 & 3.94 (3.28 to 4.73 ) & 4.48 (3.43 to 5.84$)$ & \\
\hline
\end{tabular}

*Because data on HIV were only available for cases reported between 1999 and 2005, the full multivariable analysis does not include 2006 cases. For categorical values overall $p$ values are reported.

†For cases reported between 1999 and 2005.

previous studies, ${ }^{34}$ cases with miliary disease were more likely to be co-infected with HIV. As there has been an increase in HIV co-infected tuberculosis cases in the $\mathrm{UK},{ }^{19}$ one might have expected that this explains part of the increase seen. The lack of an association between HIV co-infection and the observed rise in miliary tuberculosis could be due to the small numbers of coinfected cases, especially among south Asians. Additional reasons for this large increase remain unclear and other factors, such as increases in immunosuppressive disorders other than HIV, may have contributed to the rise. For instance, nutritional and environmental factors such as vitamin D deficiency have been associated with an increased risk of tuberculosis. ${ }^{25}$ In contrast to all extrapulmonary cases, place of birth/time since entry was not a strong risk factor for miliary disease. Miliary cases were also found to be older, which could suggest a role for reactivation disease.

Despite the large increase in miliary tuberculosis, the total number of cases remains low and comprises only around $2 \%$ of all cases each. This trend therefore does not explain the overall increase in extrapulmonary disease. Molecular epidemiological analysis may throw further light on these changing trends.

\section{Clinical implications}

Extrapulmonary cases are not infectious to others, but they are more difficult to recognise and diagnose. Clinicians need to be aware of the observed increase and have a higher index of clinical suspicion, especially among the non-UK born population. Culture is the only way to confirm the disease, and at around 53\% there is still room for improvement. Clinicians should ensure that samples from patients with suspected extrapulmonary tuberculosis in general, and from biopsy specimens in particular, are sent for mycobacterial culture. The increase in miliary tuberculosis is particularly worrisome, as cases with this form of tuberculosis are known to have particularly poor outcomes. ${ }^{3}$ 


\section{Strengths and limitations}

The analysis was based on 8 consecutive years of national surveillance data, providing a large and representative dataset. As with other observational studies, the effect of residual and unmeasured confounding cannot be excluded. One of the main limitations of the study is the inability to adjust for the effects of social risk factors, causes of immunosuppression other than HIV and time since infection. Interactions between various risk factors were found on multivariable analyses, making their effects difficult to interpret. These interactions, however, did not affect the results for the trend in extrapulmonary disease. HIV co-infection status was obtained by matching tuberculosis cases to HIV/AIDS reports, with a potential for misclassification of cases, and was not available for cases reported in 2006 and those aged $<15$ years of age, reducing the power and extrapolation of the results. As negative test results are not reported, HIV status also includes a certain extent of misclassification, and its association with extrapulmonary tuberculosis may therefore be underestimated.

\section{CONCLUSION}

In the UK the clinical presentation of tuberculosis is changing with an increasing proportion of patients now presenting with extrapulmonary manifestations. This study shows that this is related to the increasing numbers of non-UK born cases who came into the country many years before developing disease. Although the absolute numbers remain small, the increase in miliary tuberculosis was particularly large; the reasons for this remain unclear.

Clinicians need to be aware of this increasing trend and have a higher index of suspicion of extrapulmonary tuberculosis, especially among cases from the Indian subcontinent. Improvements in obtaining culture confirmation for extrapulmonary tuberculosis should be an objective.

Acknowledgements: We would like to acknowledge all those who provide information on tuberculosis and HIV/AIDS diagnoses in England and Wales. Many thanks to David Quinn for preparing the Enhanced Tuberculosis Surveillance dataset for analysis and for his role in the data matching processes and to Dr Oscar Franco for his extensive comments on this document.

Funding: No external funding was obtained.

Competing interests: None.

Ethics approval: This study was carried out with national surveillance data. The Health Protection Agency has Patient Information Advisory Group approval to hold and analyse national surveillance data for public health purposes under Section 60 of the Health and Social Care Act 2001.

The corresponding author had full access to all the data in the study and had final responsibility for the decision to submit the paper for publication.

Provenance and peer review: Not commissioned; externally peer reviewed.

\section{REFERENCES}

1. World Health Organization. Global tuberculosis control: surveillance, planning, financing. WHO Report 2008. Geneva: World Health Organization, 2008.

2. Davies PO. Clinical tuberculosis. 3rd ed. London: Arnold, 2003.

3. Sharma SK, Mohan A, Sharma A, et al. Miliary tuberculosis: new insights into an old disease. Lancet Infect Dis 2005;5:415-30.

4. National Collaborating Centre for Chronic Conditions. Tuberculosis: clinical diagnosis and management of tuberculosis, and measures for its prevention and control. London: Royal College of Physicians, 2006.

5. EuroTB, National Coordinators for Tuberculosis Surveillance in the WHO European Region. Surveillance of tuberculosis in Europe. Report on tuberculosis cases notified in 2006. Saint-Maurice, France, Institut de veille sanitaire, 2008.

6. Rieder HL, Snider DE Jr, Cauthen GM. Extrapulmonary tuberculosis in the United States. Am Rev Respir Dis 1990;141:347-51.

7. te Beek LA, van der Werf MJ, Richter C, et al. Extrapulmonary tuberculosis by nationality, The Netherlands, 1993-2001. Emerg Infect Dis 2006;12:1375-82.

8. CDC. Reported tuberculosis in the United States, 2006. Atlanta: US Department of Health and Human Services, CDC, 2007.

9. Ellis E, Dawson K, Gallant V, et al. Tuberculosis in Canada 2006, pre-release. Ottawa, Canada: Public Health Agency of Canada, 2007.

10. Erkens CGM, Kalisvaart NA, Slump E, et al. Tuberculose in Nederland 2006, surveillancerapport over de tuberculosesituatie in Nederland. Den Haag, The Netherlands: KNCV Tuberculosefonds, 2008.

11. Forssbohm M, Zwahlen M, Loddenkemper R, et al. Demographic characteristics of patients with extrapulmonary tuberculosis in Germany. Eur Respir J 2008;31:99-105.

12. Ong A, Creasman J, Hopewell PC, et al. A molecular epidemiological assessment of extrapulmonary tuberculosis in San Francisco. Clin Infect Dis 2004;38:25-31.

13. Sreeramareddy CT, Panduru KV, Verma SC, et al. Comparison of pulmonary and extrapulmonary tuberculosis in Nepal: a hospital-based retrospective study. BMC Infect Dis 2008;8:8.

14. Yang Z, Kong $Y$, Wilson $F$, et al. Identification of risk factors for extrapulmonary tuberculosis. Clin Infect Dis 2004;38:199-205.

15. Gonzalez $\mathbf{O Y}$, Adams G, Teeter LD, et al. Extra-pulmonary manifestations in a large metropolitan area with a low incidence of tuberculosis. Int J Tuberc Lung Dis 2003; 7:1178-85.

16. Cowie RL, Sharpe JW. Extra-pulmonary tuberculosis: a high frequency in the absence of HIV infection. Int J Tuberc Lung Dis 1997;1:159-62.

17. Havlir DV, Barnes PF. Tuberculosis in patients with human immunodeficiency virus infection. N Engl J Med 1999;340:367-73.

18. Anderson C, Moore J, Kruijshaar ME, et al. Tuberculosis in the UK: annual report on tuberculosis surveillance in the UK 2008. London: Health Protection Agency, 2008.

19. Ahmed AB, Abubakar I, Delpech V, et al. The growing impact of HIV infection on the epidemiology of tuberculosis in England and Wales: 1999 2003. Thorax 2007;62:672-6.

20. French CE, Glynn JR, Kruijshaar ME, et al. The association between HIV and antituberculosis drug resistance. Eur Respir J 2008;32:718-25.

21. Office of National Statistics. Ethnic Group Statistics: a guide for the collection and classification of ethnicity data. 2009. http://www.statistics.gov.uk/about/ ethnic group statistics/default.asp (accessed Sep 2008).

22. Musellim B, Erturan S, Sonmez DE, et al. Comparison of extra-pulmonary and pulmonary tuberculosis cases: factors influencing the site of reactivation. Int J Tuberc Lung Dis 2005;9:1220-3.

23. Cailhol J, Decludt B, Che D. Sociodemographic factors that contribute to the development of extrapulmonary tuberculosis were identified. J Clin Epidemiol 2005; 58:1066-71.

24. Rock RB, Olin M, Baker CA, et al. Central nervous system tuberculosis: pathogenesis and clinical aspects. Clin Microbiol Rev 2008;21:243-61.

25. Nnoaham KE, Clarke A. Low serum vitamin D levels and tuberculosis: a systematic review and meta-analysis. Int J Epidemiol 2008;37:113-9. 